\title{
Transceiver Energy Consumption Models for the Design of Low Power Wireless Sensor Networks.
}

\author{
Odey John Adinya, Li Daoliang \\ College of Information and Electrical Engineering, \\ China Agricultural University, Beijing, PRC.
}

\begin{abstract}
It is generally desired to have a wireless sensor network (WSN) that will run on little power (if possible, none at all) thereby saving cost, and the inconveniences of having to replace batteries in some difficult to access areas of usage. Despite several researches on WSN energy consumption, there is no clear transceiver energy consumption model to use when designing very low power WSN. By studying the energy consumption map of the transceiver of a WSN node in different states and within state transitions, we propose in this paper the energy consumption model of the transceiver unit of a typical sensor node. The contribution of this paper is an innovative energy consumption model based on finite automata which reveals the relationship between the aggregate energy consumption and power characteristics of the transceiver components.
\end{abstract}

Keywords- Transceiver energy model, Low power Wireless Sensor Networks.

\section{INTRODUCTION}

The emerging field of wireless sensor networks (WSN) creates a new and interesting paradigm in the way we interact with our environment. Typically, a WSN node performs several functions, including; sensing environmental physical parameters, processing the raw data locally to extract characteristic features of interest, storing this information momentarily, and using a wireless link to transmit the information to its neighbors [1], [2]. Each node in the sensor network node consists of four components (figure 1): a sensor which connects the network to physical world, computation part which consists of microcontroller or microprocessor in some application responsible for control of the sensors and a transceiver for communicating between nodes and base station, and a power supply which is usually a battery [1].

Energy consumption is a requirement for all the components of the WSN node to work, and since a wireless sensor node is typically battery operated, it is therefore energy constrained [2]. Even with the most energy dense state of the arts battery, the operational life of a miniaturized system, capable of sensing, storage and wireless telemetry, is relatively short, requiring periodic maintenance by personnel which is costly and in many cases prohibitive and/or dangerous [1].

Much work has been done on low-powered sensor nodes and their communication abilities. Some specific examples are schemes handling, the reduction of communication, effective routing and multihop schemes, the reactive partial waking up of WSN nodes [1]-[9]. Most research efforts are focused on performance comparisons and trade-off studies between various low-energy routing and self-organization protocols, [2] while keeping other system parameters fixed.
What is the power consumption at the WSN node, and how much this power consumption affects the life expectancy of such a network is one of the most fundamental questions that must be answered when designing a WSN. It is important to understand the power dissipation characteristics of the sensor node and the energy consumption metrics of the network as a whole to be able to implement a sustainable systems design.

The transceiver consumes bulk of the power available to a sensor node [1]. However previous energy consumption researches have focused on a generalized energy model without a clear indication of how the transceiver components affect the overall energy consumption of the sensor node [3]. As a result, very little has been revealed about the relationship between the transceiver power characteristics and the aggregate WSN energy consumption.

A WSN transceiver is made up of the front end, back end and assisting electronic components like Digital/Analog Converters (DACs) and Analog/Digital Converters (ADCs), Mixers, frequency synthesizers, voltage control oscillators (VCO), phase locked loops (PLL) and power amplifiers, and all these need power to work [1], [4], [12] and [13]. There are wide ranges of choices of parameters and trade-offs necessary when designing WSN node and making the optimal choices of components and design considerations go a long way to affect the energy consumption and longevity of such a network [18].

\section{PRoposed ENERgY Model.}

A transceiver can be modeled as a finite state machine (FSMs) presented as a graph in which the system's behavior is defined as finite sets of nodes (the model's states) and the links between these nodes (transitions between states).A given state reflects the evolution of the model and transitions are associated with a given logical condition or triggers to enable the execution of the transition.

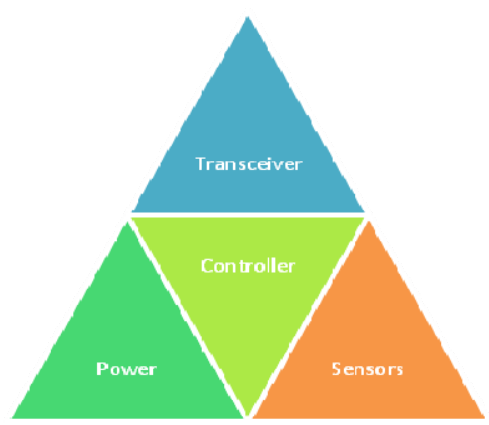

Figure 1: Components of a wireless Sensor node. 


\subsection{Mathematical Abstraction.}

A FSM is formally defined as a quintuple $(\Sigma, \mathrm{S}, \mathrm{s} 0, \delta, \mathrm{F})$, where: $\Sigma$ is the input alphabet (a finite, non-empty set of symbols). $\mathrm{S}$ is a finite, non-empty set of states, $\mathrm{s}_{0}$ is an initial state, and an element of $\mathrm{S}, \delta$ is the state-transit ion function: $\delta: \mathrm{S} \times \Sigma \rightarrow \mathrm{S}$ (deterministic finite state machine). In a nondeterministic finite machine, it would be $\delta: S \times \Sigma \longrightarrow$ $P(\mathrm{~S})$ i.e. $\delta$ would return a set of states. $\mathrm{F}$ is the set of final states, a (possibly empty) subset of S [10], [11].

We model the transceiver as a finite state machine with two basic states (active and Sleep) and a transition state between them. Our energy model presents a typical radio transceiver of four active states, a sleep state (various degrees) and twelve transition states as shown in Table 1. To model the transceiver power consumption, we have simplified the actual power consumption characteristics with the assumption that power consumption in a WSN node occurs according to a symmetric and linear function within basic states $i$ and when in transitions between two states $i$ and $j$. The energy $E_{i}$ consumed during a single visit to basic state i depends on the power consumption Pi of the underlying electronic circuitry and the time $T_{i}$ spent in that state and is modeled as

$$
\mathrm{E}_{\mathrm{i}},=\mathrm{P}_{\mathrm{i}} * \mathrm{~T}_{\mathrm{i}},
$$

\subsection{Active State.}

The active state is when the transceiver is switch on and is ready for activities. The transceiver is sending or receiving data packets or in an idle state awaiting triggers from internal or external sources.

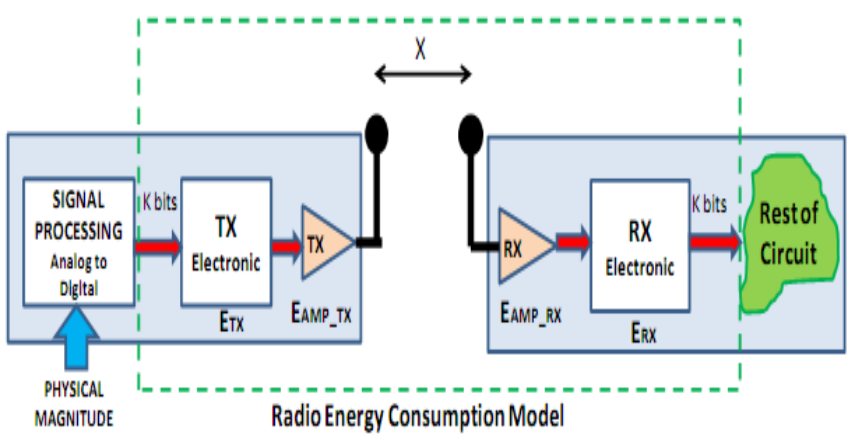

Figure 2: Block diagram of a typical transceiver [4]

\subsubsection{Transmit State.}

In transmit state; the transmitter electronics and amplifier consume large power to transmit data using the Friis free space equation in (2.6). The transceiver transmit energy consumption is modeled as $\mathrm{E}_{\mathrm{tx}}$.

$$
\mathrm{E}_{\mathrm{tx}}=\mathrm{P}_{\mathrm{tx}} * \mathrm{~T}_{\mathrm{tx}}
$$

$\mathrm{P}_{\mathrm{tx}}$ is the sum of the transmitter electronics power overhead $\mathrm{P}_{\text {overhead }}$ and amplifier $\left(\mathrm{P}_{\mathrm{A}}\right)$ at time $\mathrm{T}_{\mathrm{tx}}$.

$$
\mathrm{P}_{\mathrm{A}}=\left(\mathrm{P}_{\min }\right) /\left(\eta_{\mathrm{PA}}\right)
$$

And $\mathrm{T}_{\mathrm{tx}}$ is also defined as the ratio of the number of bits $\left(\mathrm{N}_{\mathrm{bit}}\right)$ and the bit rate $\left(\mathrm{R}_{\mathrm{bit}}\right)$. Therefore $\mathrm{E}_{\mathrm{tx}}$ can be rewritten as:

$$
\mathrm{E}_{\mathrm{tx}}=\left(\left(\mathrm{P}_{\text {overhead }}\right)+\left(\mathrm{P}_{\min } / \eta_{\mathrm{PA}}\right)\right)\left(\mathrm{N}_{\mathrm{bit}} / \mathrm{R}_{\mathrm{bit}}\right)
$$

Also

$\mathrm{P}_{\text {overhead }}=($ Header + Payload + Trailer $) /$ rate

$P_{\min }=(4 \pi f / c)^{2} \times\left(d^{n} / G r G t\right) \times R_{\text {sens }} \times L F$

$\mathrm{P}_{\min }$ is the minimum power required for communication between two nodes at a distance $\mathrm{d}$ apart using frequency $\mathrm{f}$. c is the speed of light $2.99 \times 10^{8}, \mathbf{n}$ is the path loss, Gr, Gt are the antenna gain of receiver and transmitter respectively, where $\mathbf{R}_{\text {sens }}$ is the sensitivity of the receiver and LF is loss factor. Also the Header is the length of packet header, Payload is the length of packet payload, Trailer being the length of packet trailer and rate refers to the data rate.

\section{Table 1: Transceiver States}

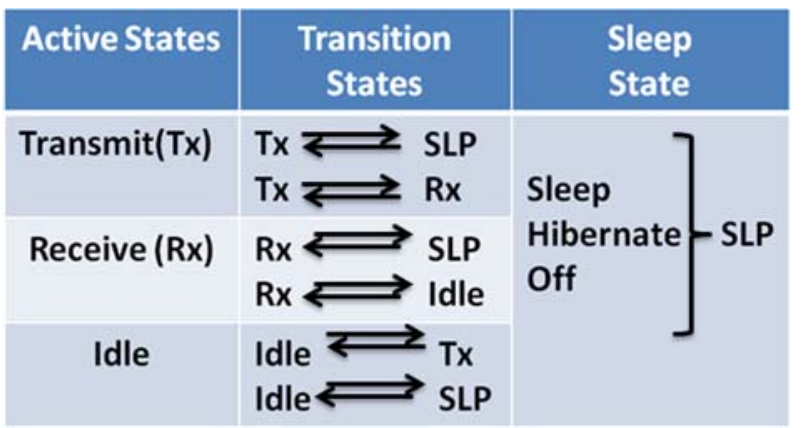

\subsubsection{Receive State.}

In this state, the receiver part of the transceiver is active and receiving data packet from the transmitter some distance away. We model the energy consumed when a receiver is active $\left(\mathrm{E}_{\mathrm{rx}}\right)$ as

$\mathrm{E}_{\mathrm{rx}}=\left(\mathrm{P}_{\mathrm{rx}} * \mathrm{~T}_{\mathrm{rx})}+\mathrm{E}_{\mathrm{decode}}\right.$

$\mathrm{P}_{\mathrm{rx}}$ and $\mathrm{T}_{\mathrm{rx}}$ are the power consumption of receiver electronic circuitry and the time duration respectively during data reception. $E_{\text {decode }}$ is the energy required by the receiver to decode nbit of data packet.

\subsubsection{Idle State.}

When a transceiver is active and ready but not currently receiving or transmitting data packets, it is said to be in an idle state. In this idle state, many parts of the transceiver circuitry are active, and others can be switched off. Most transceivers operating in idle state have power consumption almost equal to the power consumed in receive mode. The energy consumption in idle state $\mathrm{E}_{\mathrm{idle}}$ is modeled as $\mathrm{E}_{\mathrm{tx}}$ and $\mathrm{E}_{\mathrm{r}}$ but in the absence of payload overhead or decoding cost as in $\mathrm{E}_{\mathrm{tx}}$ and $\mathrm{E}_{\mathrm{rx}}$. For simplicity, we shall also model the Energy consumption of the receiver when sending beacon packets and doing clear channels assessment CCA as an idle state activity with beacon payloads overhead.

$$
\mathrm{E}_{\text {idle }}=\mathrm{P}_{\text {idle }} * \mathrm{~T}_{\text {idle }}
$$

$\mathrm{P}_{\text {idle }}$ is power used in this state listening to noise, doing a CCA scan or just nothing at time $\mathrm{T}_{\text {idle. }}$ 


\subsection{Sleep State.}

In the sleep state, a significant or all parts of the transceiver are switched off. There are transceivers offering several different sleep states, [1], [13-17]. These sleep states differ in the amount of circuitry switched off and in the associated recovery times and startup energy [3]. For example, in a complete power down of the transceiver, the startup energy include a complete initialization as well as configuration of the radio, whereas in "lighter" sleep modes, the clock driving certain transceiver parts is throttled down while configuration and operational state is remembered [1]. To get a complete energy consumption model for the transceiver, this energy consumption should also be factored in our calculation. Energy $E_{S L P}$ in sleep state is given as

$$
\mathrm{E}_{\mathrm{SLP}}=\mathrm{P}_{\mathrm{SLP}} * \mathrm{~T}_{\mathrm{SLP}}
$$

$\mathrm{P}_{\mathrm{SLP}}$ and $\mathrm{T}_{\mathrm{SLP}}$ is the power leaks of electronic circuits and time in sleep state.

\subsection{Transition States.}

The power consumption during activation and de-activation activities for transitions between states $\mathrm{i}$ and $\mathrm{j}$ are different, though our simplified model assumes $\mathrm{Pij}=\mathrm{Pji}$ and average power consumption is calculated as $\mathrm{Pij} \approx \mathrm{Pi}+\mathrm{Pj}$. The energy $\mathrm{E}_{\mathrm{SE}}$ consumed in transition state is modeled as

$$
E_{\mathrm{SE}}=\mathrm{P}_{\mathrm{ij}} * \mathrm{~T}_{\mathrm{ij}}=\mathrm{P}_{\mathrm{ji}} * \mathrm{~T}_{\mathrm{ji}}
$$

\subsection{Transceiver Energy Model.}

We present the Transceiver energy consumption as an aggregation of the energy consumption of the basic states (active and Sleep) and the transition states. From (2.4), (2.6), (2.7), (2.8) and (2.9), we present the transceiver energy consumption $\mathrm{E}_{\text {trans }}$ as

$\mathrm{E}_{\text {transc }}=\left(\mathrm{E}_{\mathrm{tx}}+\mathrm{E}_{\mathrm{rx}}+\mathrm{E}_{\mathrm{idle}}\right)+\mathrm{E}_{\mathrm{SE}}+\mathrm{E}_{\mathrm{SLP}}$

Our complete transceiver energy model is shown in Figure $\mathbf{3}$ as a finite state machine whose current state is determine by an input or trigger which enables a state transition. It models energy consumption in each basic state and transition.

III. Simulation Using Proposed Energy Model. Figure 4 shows the simulated transceiver energy consumption graph while transmitting data packets, listening, idle, receiving data packets and sleeping using our proposed model in an OPNET simulated environment. This simulated experiment evaluates the energy consumption tendency of the transceiver of a WSN node in different states using the energy consumption model abstracted in (2.12). In order to simulate and evaluate the transceiver energy model in OPNET simulation environment [17], we suppose a WSN node of five XbeeZB-pro waspmote [15] acquiring temperature data from an aquacultural environment using a Unism digital temperature $\mathrm{TH}-10507$ probe in a free space environment. The charged battery voltage (Vs) and current (Is) of Xbee- zb-pro waspmote are $4.2 \mathrm{~V}$ and $40 \mathrm{~mA}$. Supposing the switch energy consumption of TH10507 are eoff-on = $0.0002 \mathrm{~J}$ and eon-off $=0.0001 \mathrm{~J}$. We are assuming the energy consumption by the processor; sensor and other units remain constant within the duration of this simulated experiment. The node uses the random routing mode in OPNET and the AODV routing protocol at a simulation distance of $100 \mathrm{~m}$ at a total time length of $200 \mathrm{~s}$.

Acquired temperature data at the of $250 \mathrm{kbps}$ is transmitted between nodes and the time and energy required to do a transmit, receive, sleep, stay idle and do CCA scan was captured (Table 2).

In our experiment, we separated the energy consumption of the transmitter electronics from the energy consumption of the transmitter during data transmit to emphasize the nominal contribution of the transceiver electronic components to the transceiver aggregate energy consumption map. In the result of this simulated experiment as shown in Table 2 and Figure 4, the largest energy consumption is by electronic components of the transceiver, followed by the receive state. The power consumption by the electronic components like amplifiers, filters, ADC, DAC grows exponential with time therefore having the greatest impact on the energy trend curve. The energy consumption is lower in the idle states and transition states and is lowest during the Sleep state.

From equation 2.1, we had expected a linear and symmetric relationship between power consumed and the duration of activity of the transceiver. However in our simulated experiment, there appears to be an inflection in the energy consumption curve of the various states at $140 \mathrm{~ms}$. This is possibly as a result of attenuation of signal through the medium of communication (air), consequently an increase in power uptake at the various states to compensate for path loss. Validation of this interesting spike in power consumption will form the subject of future and further research not covered in this paper.

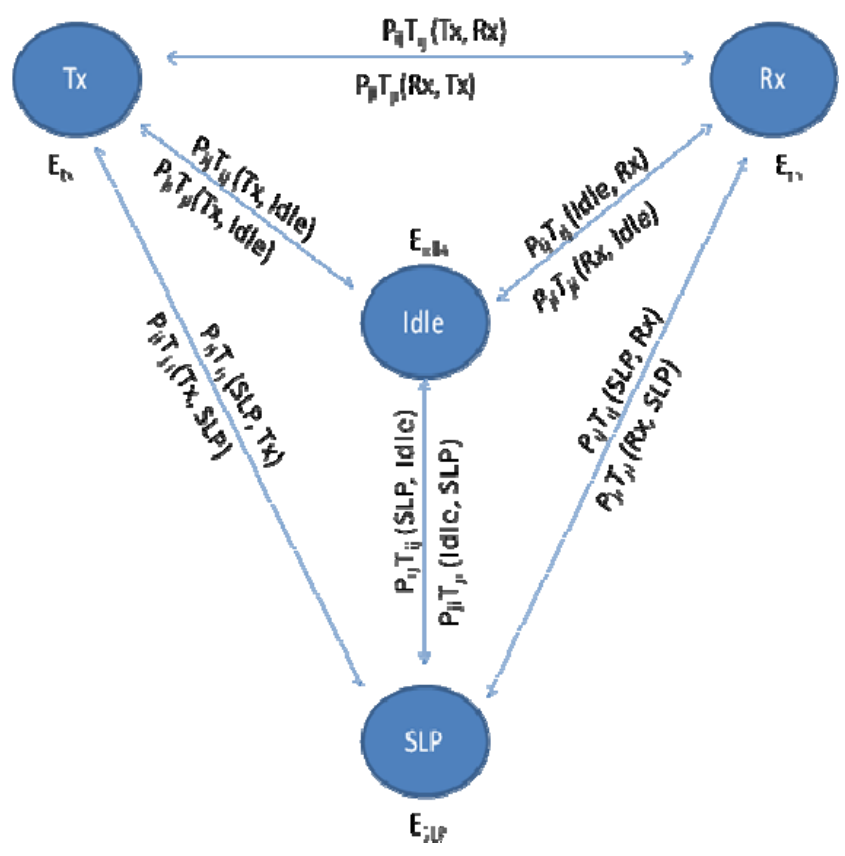

Figure 3: Complete Transceiver Energy Model. 
Table 2: Experimental results of Energy model.

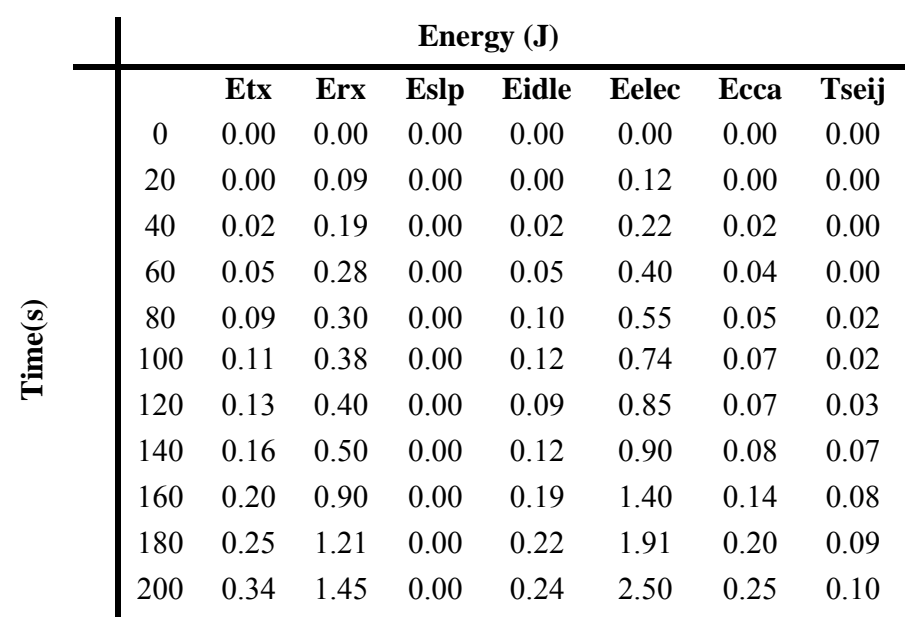

Table 3: XBee modules in waspmotes from Libelium [15]

\begin{tabular}{l|ccccc}
\multicolumn{1}{c|}{ Model } & Protocol & Frequency & txPower & Sensitivity & Range \\
\hline Xbee-802.15.4 & 802.15 .4 & $2.4 \mathrm{Ghz}$ & $1 \mathrm{~mW}$ & $-92 \mathrm{~dB}$ & $500 \mathrm{~m}$ \\
Xbee-802.15.4Pro & 802.15 .4 & $2.4 \mathrm{Ghz}$ & $100 \mathrm{~mW}$ & $-100 \mathrm{dBm}$ & $7000 \mathrm{~m}$ \\
Xbee-ZB & ZigBee-Pro & $2.4 \mathrm{Ghz}$ & $2 \mathrm{~mW}$ & $-96 \mathrm{dBm}$ & $500 \mathrm{~m}$ \\
Xbee-ZBPro & ZigBee-Pro & $2.4 \mathrm{Ghz}$ & $50 \mathrm{~mW}$ & $-102 \mathrm{dBm}$ & $7000 \mathrm{~m}$ \\
Xbee-868 & RF & $868 \mathrm{Mhz}$ & $315 \mathrm{~mW}$ & $-112 \mathrm{dBm}$ & $12 \mathrm{~km}$ \\
Xbee-900 & RF & $900 \mathrm{Mhz}$ & $50 \mathrm{~mW}$ & $-100 \mathrm{dBm}$ & $10 \mathrm{~km}$ \\
Xbee-XSC & RF & $900 \mathrm{Mhz}$ & $100 \mathrm{~mW}$ & $-106 \mathrm{dBm}$ & $12 \mathrm{~km}$
\end{tabular}

Table 3 shows the frequencies and communications ranges of the Libelium Waspmote models. The communication range or distance will be greatly extended when using lower frequencies due to low path loss attenuation. With low path propagation loss, the antenna gain would not become an important factor in the system link budget. Communication using lower frequencies would be an ideal choice if the density of deployment of a WSN allows for some short distances apart between nodes.

Multi-hops, short-range inter nodal communication can also be adopted so that more nodes can be in the sleep state to reduce power consumption as this also decrease the single-hop communication distance. From equation (2.1)(2.9), figure 3 and table 2 , the range, frequency of transmission, antenna characteristics (sensitivity and gains), modulation and demodulation scheme, routing and MAC protocols, topology control, loss factor and data rate play important role towards the aggregate energy consumption map of a transceiver. This makes these parameters very important factors for consideration when designing a low power WSN.

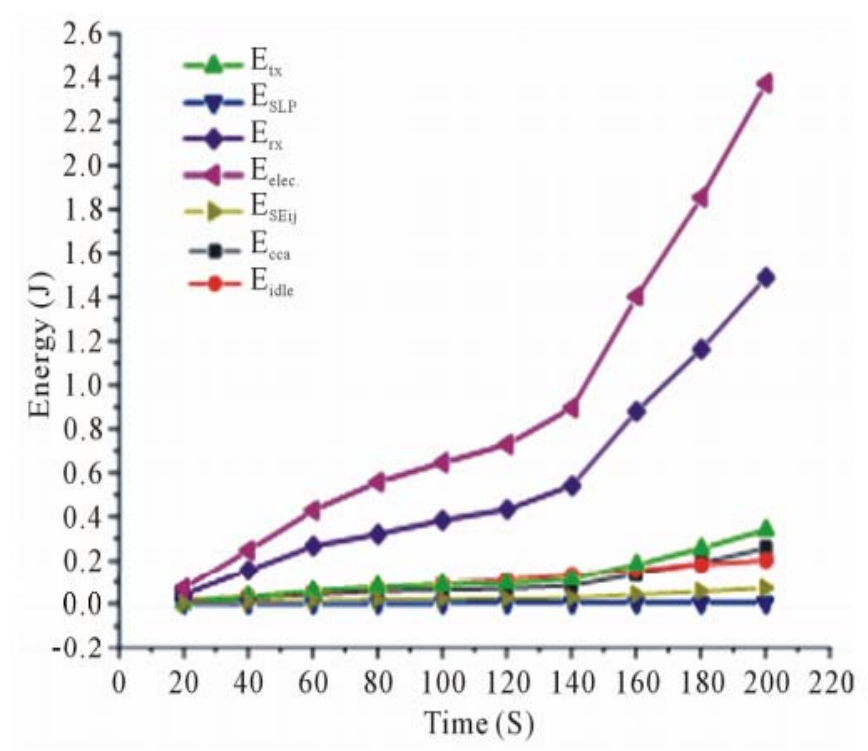

Figure 4. Transceiver States energy consumption. 


\section{CONCLUSION}

Energy consumption as discussed in this paper is a precious resource in wireless sensor networks. Considerable energy efficiency should make an evident optimization goal and be carefully distinguished to form actual, measurable figures of merit.

In Section 2, we propose and abstracted our Transceiver Energy model using finite automata. Section 3 focuses on a simulation of a transceiver energy model using our energy model. Understanding and applying the transceiver energy consumption models abstracted here will enable design of an efficient and low power WSN as energy consumption parameters are often inter related with performance and other user expectations. Further evaluation of this energy model on other IEEE 802 transceiver technologies should yield interesting results in future research.

\section{REFERENCES}

[1] Holger Karl, and Andreas Willig,"'Protocols and Architectures for Wireless Sensor Networks. John Wiley \& Sons, 2005, pp. 15-329.

[2] C.-Y. Chong and S. Kumar,"Sensor networks: Evolution, opportunities, and challenges", Proc. IEEE, Vol. 91, No. 8, pp.12471256, 2003.

[3] I.F. Akyildiz et al., "Wireless Sensor Networks: A Survey," Computer Networks, vol. 38, 2002, pp. 393-42.

[4] Sandra Sendra, Jaime Lloret, Miguel García and José F. Toledo, "Power saving and energy optimization techniques for Wireless Sensor Networks", Journal of communications, Vol 6. No 6, Sept, 2011

[5] A. Chandrakasan, R.Min, M. Bhardwaj, S-H Cho, and A.Wang, "Power Aware Wireless Microsensor Systems," ESSCIRC, Florence, Italy, September 2002.

[6] Ali Norouzi and Ahmet Sertbas, "An Integrated Survey in Efficient Energy Management for WSN using Architecture Approach“, Int. J.
Advanced Networking and Applications Volume; 03, Issue; 01, Pages: 968-977 (2011).

[7] Vikas Kumar and Rajender Kumar" Low Power Wake up Receiver for Wireless Sensor Network"; IJCST Vol. 1, Issue 1, Sept. 2010.

[8] A Roy and N Sarma, "Energy Saving in MAC Layer of Wireless Sensor Networks: a Survey"; National Workshop in Design and Analysis of Algorithm (NWDAA)", Tezpur University, India, 2010.

[9] Jerker Delsing, John Borg, Jonny Johansson" Architecture for Extreme Low Power Sensing in Wireless Sensor Network Devices": The Fifth International Conference on Sensor Technologies and Applications, SENSORCOMM 2011.

[10] Wikipedia, "Finite State Machines". http://en.wikipedia.org/wiki/finite automata. Accessed Dece-mber, 2011.

[11] James A. Anderson and Thomas J. Head." Automata theory with modern applications." Cambridge University Press. pp. 105-108. (2006), ISBN 9780521848879.

[12] Q. Yang, X. H. Chen and J. H. Shi, "Low Power Design of the Terminal Node for Wireless Sensor Network," Journal of Xiamen University (Natural Science), Vol. 47, No. 3, 2008, pp. 357-358.

[13] X. F. Wang, J. Xiang and B. J. Hu, "Evaluation and Improvement of an Energy Model for Wireless Sensor Networks," Chinese Journal of Sensors and Actuators, Vol. 22, No. 9, 2009, pp. 1319-1321.

[14] J. Moreno Molina, J. Haase, and C. Grimm. "Energy consumption estimation and profiling in wireless sensor networks. In ARCS '10 23th International Conference on Architecture of Computing Systems 2010 Workshop Proceedings, pages 259-264, Feb. 2010

[15] Libelium Corporation, "Waspmote Technical guide". http://www.libelium.com /documentation/waspmote/-waspmotetechnical_guide_eng.pdf. Accessed Dece-mber, 2011.

[16] J. Moreno Molina, J. Haase, C. Grimm and J. Wenninger "Energy Profiling Technique for Network-Level Energy Optimization" IEEE Africon 2011 - The Falls Resort and Conference Centre, Livingstone, Zambia, 13 - 15 September 2011.

[17] B. Han, D. Z. Zhang and T. Yang, "Energy Consumption Analysis and Energy Management Strategy for Sensor Node,"International Conference on Information and Automation, Proceedings of the 2008 IEEE, Vol. 6, 2008, pp. 211-214.

[18] X. Li, "Network Modeling and Simulation with OPNET Modeler," Xidian University Press, Xi'an, 2006, pp.1-15. 